\title{
AN EXAMPLE IN FIXED POINT THEORY
}

\author{
GORDON G. JOHNSON
}

\begin{abstract}
An example is given of a homeomorphism of $R^{2}$ onto $R^{2}$ which has no fixed points, such that each of its iterates has a fixed point.
\end{abstract}

A few years ago A. Granas posed the question: Does there exist a compact metric space $X$ and a mapping (i.e. continuous function) $f: X \rightarrow X$ with no fixed points, such that each iterate $f^{n}(n \geqq 2)$ of $f$ has a fixed point?

W. Kuperberg has given as a simple solution (unpublished) the following: $X$ is the set of all points on the Cartesian plane whose polar coordinates $(p, \theta)$ satisfy the inequality $1 \leqq p \leqq 2$ and $f: X \rightarrow X$ by

$$
f(p, \theta)=(p, \theta+(2+p) \pi / 3) .
$$

It is easy to see that $f$ is a homeomorphism of $X$ onto $X$ and that $f$ has no fixed points. Oberve also that if $n$ is an integer greater than 1 , then any point on the circle $p=p_{n}$ is a fixed point for $f^{n}$ where $p_{n}=6 K(n) / n-2$ and $K(n)$ is an integer such that $\frac{1}{2} \leqq K(n) / n \leqq \frac{2}{3}$.

Our example gives an affirmative answer to the question: Is there a homeomorphism $h$ of the Cartesian plane $\pi$ onto itself which has no fixed point such that each iterate $h^{n}(n \geqq 2)$ of $h$ has a fixed point?

To this end, define the homeomorphism $g$ from $\pi$ onto $\pi$ by $g(x, y)=$ $(-x, y)$, let $W=\{(x, y):|x| \leqq 8\}$, and define $f$ on $\pi-W$ to be the identity function.

We now show how to define $f$ on $W$ such that $h=f \circ g$ is a homeomorphism of $\pi$ onto $\pi$ with the desired properties.

As an aid to this end, consider Figures 1 through 5.

In Figure 2 we indicate the image of a typical interval $[a, h]$ under $f$.

In Figure 3 we note that we require $c \rightarrow c^{\prime}$, in Figure 4 that $b \rightarrow b^{\prime}$, and in Figure 5 that $c$ maps onto $c^{\prime}$. These are the essential actions of $f$ on $W$.

In Figure 1 we indicate the action of $f$ on a portion of $W$ and note that $h^{3}\left(P_{3}\right)=P_{3}, h^{4}\left(P_{4}\right)=P_{4}$ and $h^{5}\left(P_{5}\right)=P_{5}$.

Presented to the Society, November 16, 1973; received by the editors July 11, 1973. AMS (MOS) subject classifications (1970). Primary 54C20, 55A05.

Key words and phrases. Fixed point, iterations, homeomorphisms.

(c) American Mathematical Society 1974 


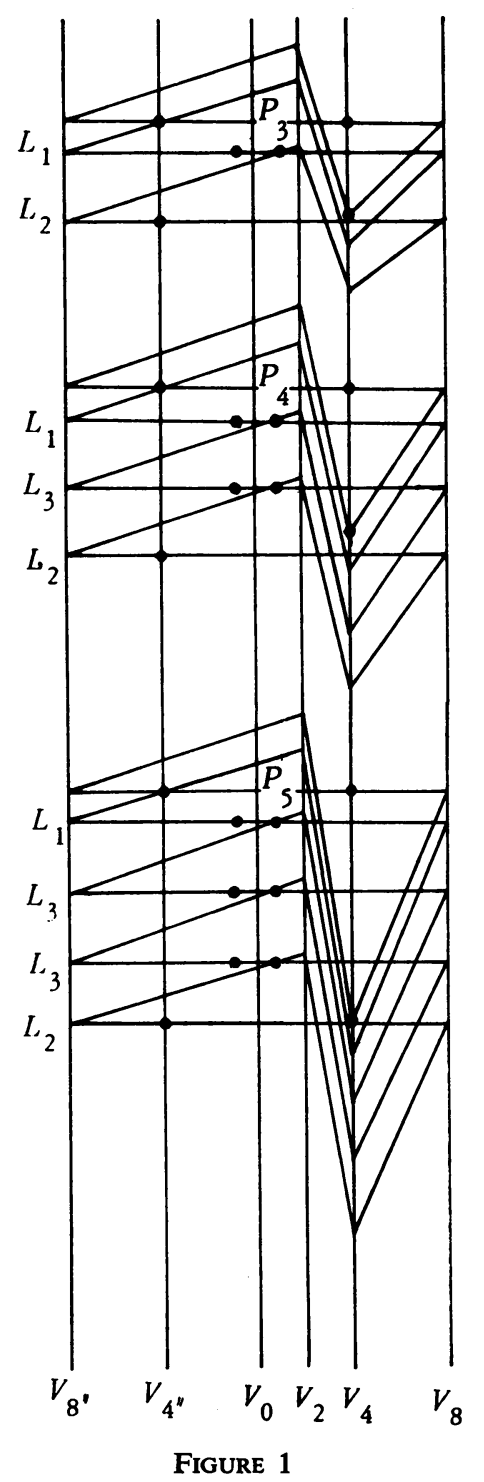




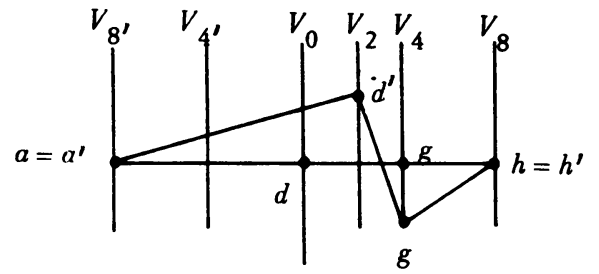

FIGURE 2

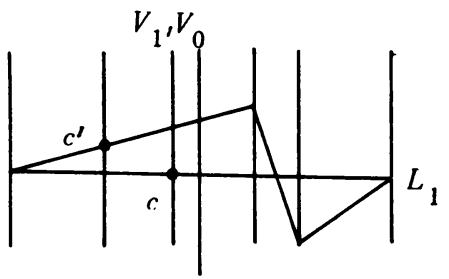

FIGURE 3

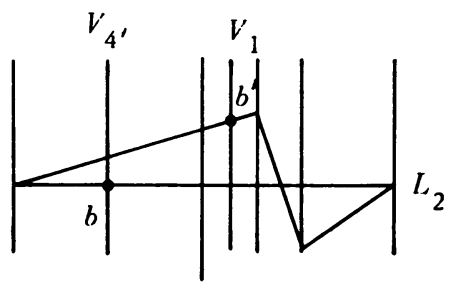

FIGURE 4 


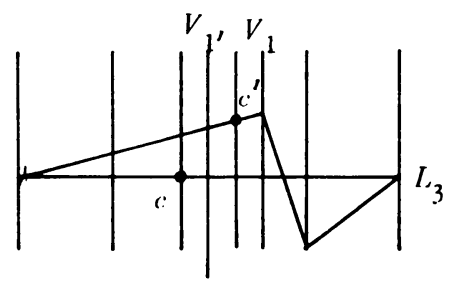

Figure 5

It is clear that $f$ can be defined on $W$ such that $h=f \circ g$ has the desired properties.

Department of Mathematics, University of Houston, Houston, Texas 77004 